\title{
Evaluation and Management of Alcohol use Disorder among Older Adults
}

\author{
Pallavi Joshi $^{1} \cdot$ Karen T Duong ${ }^{2} \cdot$ Louis A. Trevisan $^{1} \cdot$ Kirsten M. Wilkins ${ }^{1}$ (D) \\ Accepted: 27 April 2021 / Published online: 24 July 2021 \\ (C) The Author(s), under exclusive licence to Springer Science+Business Media, LLC, part of Springer Nature 2021
}

\begin{abstract}
Purpose of Review The prevalence of alcohol use disorder (AUD) among older adults in the United States is rising, but remains underdiagnosed, underreported, and inadequately managed. This review highlights the medical, social, and cultural factors of AUD in older adults and provides guidelines for its screening, evaluation, and management.

Recent Findings The COVID-19 pandemic has created additional challenges and barriers to care, as older adults may have disproportionate worsening of anxiety, depression, and substance use resulting from increased isolation related to physical distancing and shelter-in-place guidelines.

Summary All older adults should be routinely screened for AUD with standardized screening tools. If a patient's screening results are positive, a clinician should conduct a brief assessment, which may be supplemented by laboratory tests. Most older adults at risk for alcohol misuse do not need specialized SUD treatment, but most can benefit from Screening, Brief Intervention, and Referral to Treatment (SBIRT) to prevent substance misuse before it occurs. Medications for the treatment of AUD in older adults include naltrexone, acamprosate, disulfiram, gabapentin and topiramate. Psychosocial treatments, including mutual help groups, are equally important.
\end{abstract}

Keywords Geriatrics $\cdot$ Alcohol use disorder $\cdot$ Older adults $\cdot$ Substance use disorders

\section{Introduction}

The prevalence of substance use disorders (SUD) among older adults is rising in the United States and alcohol is the most commonly used substance in this population. The 2019 National Survey on Drug Use and Health (NSDUH) found that approximately 5.6 million (10.7\%) adults aged 65 years and older engaged in past-month binge alcohol use, and an estimated 1.5 million (2.8\%) engaged in past-month heavy alcohol use [1]. In the 2012-2013 Wave of the National Epidemiologic Survey on Alcohol and Related Conditions (NESARC III), 2.3\% of adults aged 65 years and older met criteria for Alcohol Use Disorder (AUD) within the past 12 months, and $13.4 \%$ met criteria during their lifetime [2].

This article is part of the Topical Collection on Geriatric Psychiatry

Kirsten M. Wilkins

kirsten.wilkins@yale.edu

1 Department of Psychiatry, Yale School of Medicine, 300 George Street, New Haven, CT 06511, USA

2 Department of Psychiatry, University of Texas Southwestern Medical Center, 5323 Harry Hines Blvd, Dallas, TX 75390, USA
The increase in SUD among older adults is driven in part by the aging Baby Boomer population, who have higher rates of earlier life substance use and increased later life prevalence compared to previous geriatric cohorts. Proposed theories for the higher numbers of SUD among Baby Boomers include: (a) higher population numbers relative to previous cohorts; (b) increased life expectancy which allows older adults to use substances for longer; (c) generational attitudes including desire for personal gratification and greater acceptance of drug use; (d) greater exposure to drugs and higher rates of use when younger $[3,4 \bullet]$.

Older adults are not a homogenous group, however, and their presentations may vary based on medical comorbidities, lifestyle factors, and duration and severity of substance use. Additionally, they may have a range of opinions and attitudes towards substance use; thus, an age and culturally sensitive and individualized approach can improve treatment outcomes [3]. Chronological age may not reliably predict health status. Some adults over 65 years of age may have better physical health status than their similarly aged peers. On the other hand, chronic alcohol use contributes to increased morbidity, exacerbation of existing health conditions, and neurotoxic and 
neuropathological changes [5]. Despite this growing problem, AUD in later life is often underdiagnosed, underreported, or overlooked, and consequently is often not managed [4•]. Diagnostic and Statistical Manual of Mental Disorders (DSM) criteria are geared towards younger adults and may not appropriately screen and diagnose older adults. Social and occupational impairments due to alcohol use may go unnoticed or unreported for older adults who are retired, live alone, or are socially isolated [4•]. DSM criteria highlight increasing use and efforts to cut back on use. However, many older adults continue with problematic drinking at the same level as they have for years and may have problematic use without efforts to cut back and at lower levels of use compared to younger adults [6]. Additionally, AUD in older adults can mimic other diagnoses which are common in later life, such as cognitive impairment or depression. Ageist beliefs among patients, family members and providers pose additional barriers to diagnosis and treatment. Older adults may minimize their substance use to healthcare providers. Family members may believe that SUDs in late life do not exist or do not need treatment, and consequently overlook substance use among older adults. Finally, healthcare providers may refrain from asking about substance use due to fear of offending older adults or may focus more on their reports of physical complaints [7].

The COVID-19 pandemic has created additional challenges for older adults, who are especially vulnerable to the detrimental effects of isolation, and may have disproportionate worsening of anxiety, depression, and substance use triggered by physical distancing and shelter-in-place guidelines [8•]. In a self-report survey of 1982 American adults, 34\% reported binge drinking during the COVID-19 pandemic. For every week spent at home, there was a 1.21 greater odds of binge drinking (95\% CI: 1.08-1.35), and binge drinkers with previous or current depression had greater increase in alcohol consumption compared to those without depression (AOR 1.80, 95\% CI: 1.15-2.81) [9]. A study of 3358 middle-aged British adults found that the prevalence of highrisk drinking increased from $19.4 \%$ between 2016 and 2018 (when the cohort was aged $46-48$ years) to $24.6 \%$ in May 2020 (aged 50 years) and the prevalence of drinking $\geq 4$ times a week doubled from $12.5 \%$ to $26 \%$ during this period [10]. This increase was not moderated by gender, marital status, education level, or presence of a chronic illness. Barriers to SUD care during COVID-19 include the closure of treatment facilities, focus of emergency departments on COVID19 patients, physical distancing and shelter in place orders affecting mental health, and threats to income and resources of individuals with SUDs [11]. Older adults are especially vulnerable to the physical and mental health challenges and barriers to SUD care imposed by the intersection of the COVID pandemic and the SUD epidemic.
Evaluation for Alcohol Use Disorder in Older Adults

Alcohol affects older adults differently than younger adults due to age-related physical changes including decreased lean body mass and total body water, decreased liver metabolism, and increased blood-brain barrier permeability and neuronal sensitivity to alcohol [1]. Consequently, older adults have higher blood alcohol concentrations and increased impairment compared to younger adults with equal levels of consumption. Older adults are more likely to be taking multiple medications which increases their risk for drug-drug interaction and falls. Finally, continued alcohol use in late life increases the risk of falls, cognitive impairment, heart disease, hypertension, and hepatitis $[12,13]$. Despite these risks, older adults are less likely than younger adults to be screened and assessed for substance misuse [1].

\section{Screening}

The Substance Abuse and Mental Health Service Administration (SAMHSA) Treatment Improvement Protocol (TIP) consensus panel recommends that all older adults be screened for alcohol, tobacco, prescription drug, and illicit drug use at least annually [14•]. Establishing a thorough history of substance use can help providers identify possible substance misuse or concerns and provide an opportunity for education and prevention for older adults with low or absent substance use. Screening can lead to earlier treatment and improved health [12].

The U.S. Preventive Services Task Force (USPSTF) recommends screening older adults for alcohol misuse and brief counseling for those who misuse alcohol [15]. A variety of validated screening tools are available (Table 1). A systematic review of 9 studies that included 6353 patients found that the Alcohol Use Disorders Identification Test (AUDIT) was useful for screening for hazardous alcohol use in patients older than 60 years, and that the CAGE questionnaire was helpful in screening for alcohol dependence [16].

\section{CAGE}

The CAGE (Cut down, Annoyed, Guilty, Eye opener) Questionnaire is a 4-item questionnaire that asks about lifetime alcohol use. A positive response to any of the 4 questions is concerning for alcohol misuse [17]. Although the CAGE questionnaire is quick and frequently used, it is not as reliable in detecting problematic use in older adults because it does not probe for binge drinking, which is a highly prevalent form of alcohol misuse in older adults. 
Table 1 Screening Tools for Alcohol Use Disorder in Older Adults

\begin{tabular}{lllll} 
Screening Tool & Current or Lifetime Use & Score Range & Score Indicating Need for Comprehensive Evaluation & Self- or Provider-Administered \\
\hline CAGE $^{1}$ & Lifetime & $0-4$ & $\geq 1$ & Provider \\
AUDIT $^{2}$ & Past year & $0-40$ & $\geq 5$ & Provider \\
AUDIT-C $^{3}$ & Past year & $0-12$ & Women: $>3$ & Provider \\
SAMI $^{4}$ & Past few months & $0-5$ & Men: $>4$ & Self \\
SMAST-G $^{5}$ & Lifetime & $0-10$ & $\geq 2$ & Self \\
\hline
\end{tabular}

${ }^{1} \mathrm{CAGE}=$ Cut down, Annoyed, Guilt, Eye-Opener [17]

${ }^{2}$ AUDIT $=$ Alcohol Use Disorders Identification Test [21]

${ }^{3}$ AUDIT-C $=$ Alcohol Use Disorders Identification Test-Consumption [22]

${ }^{4}$ SAMI $=$ Senior Alcohol Misuse Indicator [20]

${ }^{5}$ SMAST-G $=$ The Short Michigan Alcoholism Screening Test-Geriatric Version [24]

\section{AUDIT and AUDIT-C}

The Alcohol Use Disorders Identification Test (AUDIT) was developed to screen for heavy alcohol use [18]. It has been shown to have reliability in AUD screening and can identify alcohol misuse in adults aged 65 and older [19,20]. The cutoff score indicating hazardous and harmful alcohol use for the AUDIT is 8; however, for older adults, a score of 5 indicates a need for further assessment [21]. The AUDIT-C is a shorter version of the AUDIT containing only three questions [22].

\section{SAMI}

Senior Alcohol Misuse Indicator (SAMI) is a five-item questionnaire which includes a checklist of symptoms and openended questions about alcohol use [20]. A score $\geq 1$ suggests alcohol misuse in older adults [23].

\section{SMAST-G}

The Short Michigan Alcoholism Screening Test-Geriatric Version (SMAST-G) is the first brief alcohol screening tool developed for older adults [24]. A positive response to two or more items suggests potential alcohol misuse [1].

\section{Assessment}

If a patient's screening results are positive, a clinician should conduct a brief assessment, including asking about the frequency and severity of alcohol use, the type and frequency of problems due to use, and physical and mental factors impacted by alcohol use. A complete substance use assessment includes full mental health, medical, family, vocational, social, sexual, financial, legal, substance use, and SUD treatment histories. A health history and physical exam should be conducted to identify common co-occurring conditions such as sleep disturbances and chronic pain that may be suggestive of substance misuse. The history and physical exam can be supplemented by basic metabolic tests, liver function and electrolyte tests, urine toxicology screens, breath alcohol testing, and laboratory tests such as gamma-glutamyltransferase (GGT), carbohydrate-deficient transferrin (CDT) and ethyl glucuronide (ETG) to quantify recent or underreported alcohol use. A physical health assessment and laboratory workup can identify medical problems related to substance use that may need treatment, as well as identify comorbidities such as liver disease and potential drug-drug interactions that can determine choice of treatment.

\section{Management of Alcohol use Disorder in Older Adults}

Most older adults at risk for alcohol misuse do not need specialized SUD treatment. However, most can benefit from Screening, Brief Intervention, and Referral to Treatment (SBIRT) to prevent substance misuse before it occurs. SBIRT approaches include screening for possible alcohol misuse and level of risk, offering a brief outpatient intervention to help patients understand the need to change their alcohol misuse, and referrals to SUD treatment programs for patients who need more specialized assessment or intervention. Healthcare providers can easily incorporate SBIRT into standard practices. Current research shows that brief interventions can reduce alcohol consumption and substance misuse among older adults $[25,26]$.

Brief alcohol interventions (BAIs) consist of one or more time-limited conversations with patients to help reduce unhealthy alcohol use. The goal is to implement harm reduction rather than promoting abstinence only. Implementing psychoeducation can include discussing the National Institute on Alcohol Abuse and Alcoholism's recommended 
daily drinking limits (i.e., adults age 65 and older should not consume more than seven drinks in a week and more than three drinks on a given day) [27]. Other psychoeducation can include discussion of medical consequences of alcohol use such as cognitive impairment, increased risk of falls, and liver dysfunction/disease. Motivational interviewing techniques are commonly used in BAIs and can be administered not only by a physician, but also other members of the treatment team such as nurses, social workers, and psychologists. Historically, BAIs have often been delivered as part of the SBIRT model, and thus are seen commonly in the primary care setting [28].

Treatment planning should involve shared decision making to choose the level of care that is the least intensive but will still address the patient's needs. Depending on the patient's needs and treatment goals, treatment may be able to be provided in a primary care or general mental health setting; alternately, referral to specialty treatment may be indicated. An individualized assessment will help determine what level of care is indicated. Factors shown to improve patient outcomes include shared decision making with the patient and incorporation of the older adult in treatment programs where other members are of similar age and functional status [29-31]. Treatment planning for the older adult should also include consideration of age-related factors such as cognition, hearing impairment, or any other physical or functional decline [14•]. If cognitive impairment is present, the extent of the patient's impairment will need to be assessed and treatment planning may need to involve family members or legal guardians [32, 33].

If the patient requires detoxification, the optimal setting for safe withdrawal will need to be considered (i.e., outpatient or inpatient). If the patient does not have access to addictionspecific treatment and does not require medically supervised withdrawal, the patient may be a candidate for outpatient detoxification in the general health care setting [14•]. However, outpatient detoxification is less commonly employed in older adults given medical comorbidities. Inpatient detoxification is recommended if the patient requires medically supervised withdrawal which includes routine monitoring of vitals and withdrawal symptoms using the Clinical Institute Withdrawal Assessment of Alcohol Scale-Revised (CIWA-Ar) and administration of medications such as benzodiazepines [34]. Of note, older adults are at higher risk of developing delirium, having protracted withdrawal, and having worsening medical conditions as compared to their younger counterparts [35]. Acute inpatient treatment may be limited to medically supervised withdrawal followed by a step-down level of care to an intensive outpatient program (IOP), residential recoveryoriented rehabilitation program, or outpatient clinic [14•, 34].

Other co-occurring psychiatric illness can often be seen in conjunction with alcohol use disorder. When active, untreated symptoms of a co-occurring psychiatric illness are present, treatment of the underlying psychiatric illness should also be addressed; successful addiction treatment is more likely if these conditions are stabilized [14•]. Consultation with geriatric and/or addiction psychiatry specialists is recommended for older adults with co-morbid AUD and psychiatric illness.

Once the patient has completed any necessary steps for medical stabilization including outpatient or inpatient detoxification and has been able to consistently abstain from alcohol use, they may need ongoing assistance with recovery management support. Patients that are more likely to need ongoing recovery support may include patients with co-occurring medical conditions or psychiatric disorders and individuals with limited social support [36].

Figure 1 depicts a recommended algorithm for the screening and treatment of alcohol use disorders among older adults.

\section{Maintenance Treatment for Alcohol Use Disorders: Pharmacologic Interventions}

The SAMHSA consensus panel recommends using medications to treat alcohol use disorder in older adults when necessary. Situations that may necessitate medications include patients in whom psychosocial interventions have not been successful and/or who continue to struggle with cravings and return to alcohol use. Factors to consider when prescribing for older adults include evaluating for potentially harmful drug-drug interactions, using lower doses of medications, ensuring medication adherence, and ensuring recovery supports [14•]. Currently there are three medications with FDA indication to treat alcohol use disorder which include naltrexone, acamprosate, and disulfiram. Two additional medications with off label use for alcohol use disorder are gabapentin and topiramate [37]. None of these medications are specifically contraindicated for use in older adults, but caution should be exercised when using these pharmacologic agents in this population (Table 2).

\section{Naltrexone}

Naltrexone is an opiate receptor blocker that reduces cravings for alcohol and decreases the rate of return to heavy drinking. There have been some studies that suggest naltrexone is tolerable in adults ages 50 and older, but widespread data about tolerability in older populations are lacking [38]. Patients who are on or require prescription opioids for pain relief are not good candidates for naltrexone as naltrexone may cause significant opioid withdrawal symptoms [14•].

\section{Disulfiram}

Disulfiram works by inhibiting aldehyde dehydrogenase which triggers an acute physical reaction to alcohol. This 
Fig.1 Screening and treatment algorithm for alcohol use in older adults

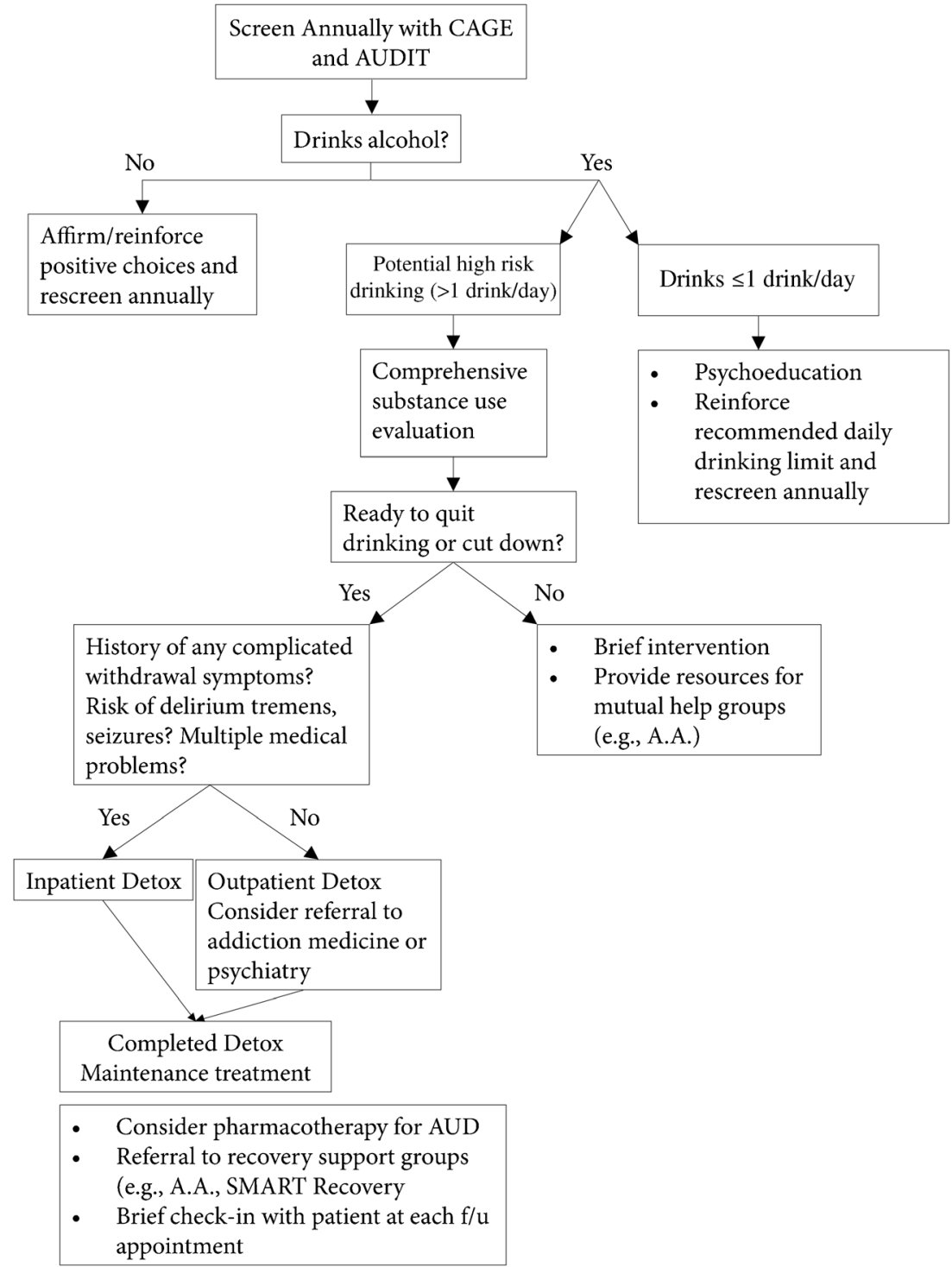

reaction includes tachycardia, flushing, nausea, chest pain, dizziness, and blood pressure changes [39, 40]. The physical reaction of disulfiram and alcohol can be harmful in older adults which makes it less recommended in the geriatric population [41, 42]. Furthermore, use of disulfiram requires patients adhering to strict medication protocols and monitoring of compliance [43]. Disulfiram should be used with caution in older adults due to higher rates of medical comorbidities, but as always, the decision to initiate depends on the overall risk/benefit ratio.

\section{Acamprosate}

Acamprosate can support abstinence by reducing cravings and the pleasurable effects associated with alcohol [1, 44]. It can also help lessen symptoms related to abstinence such as insomnia and anxiety [39]. It is the preferred agent in patients with hepatic impairment [45]. There is limited research on acamprosate use specifically in older adults, but kidney function should be taken into consideration before starting this medication. Given that older adults are at higher risk of renal impairment, baseline renal function and frequent monitoring of renal function should accompany treatment with acamprosate $[14 \bullet]$.

\section{Gabapentin}

Gabapentin is FDA-approved for post-herpetic neuralgia and adjunctive therapy for focal (partial) seizures. This medication can be used off-label for alcohol use disorder, as studies have found it to help with mild alcohol withdrawal syndrome and neuropathic pain [46, 47]. 


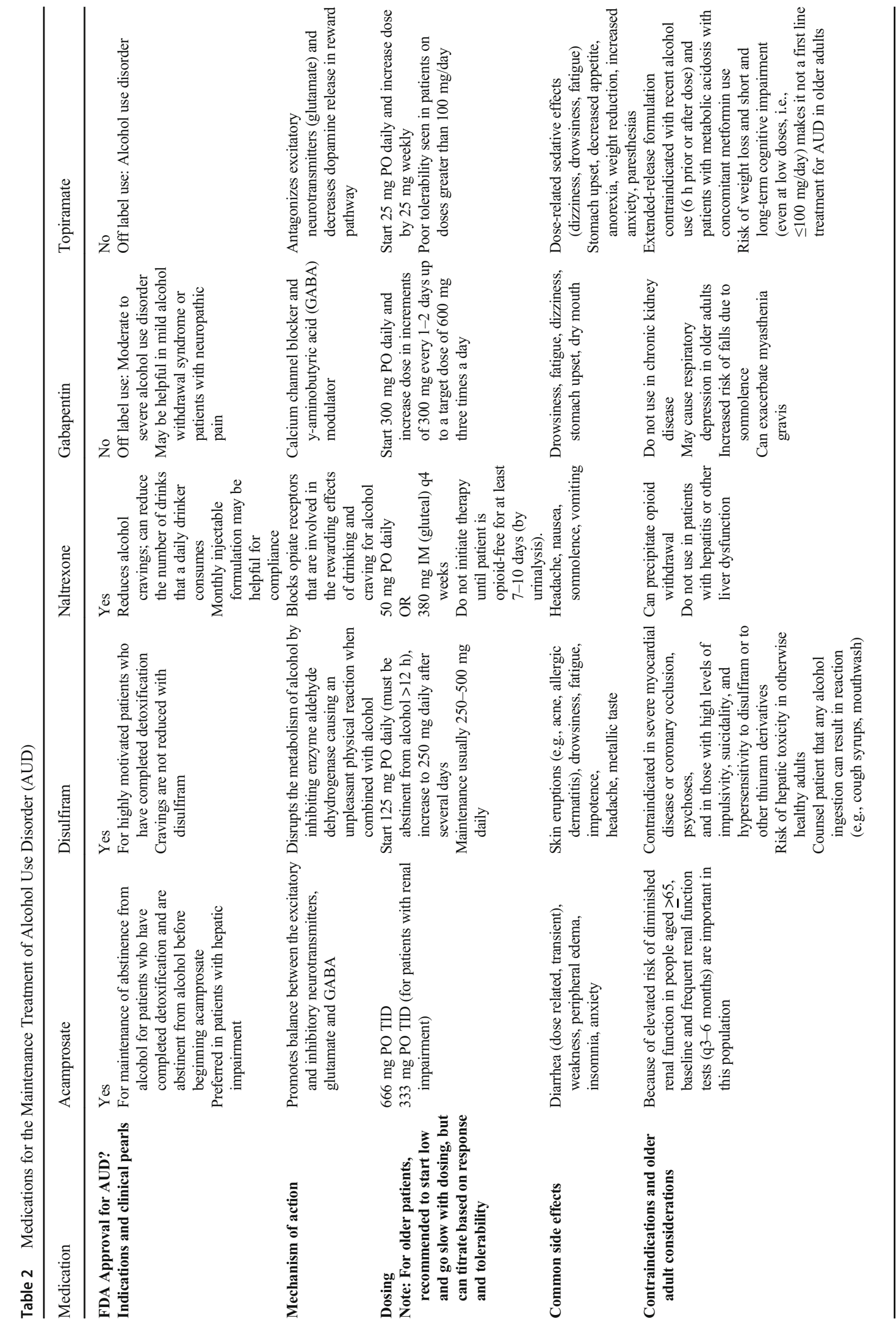




\section{Topiramate}

Topiramate is an anticonvulsant that has FDA indications for migraine prevention and seizures. It can be used off label in alcohol use disorder for cravings and withdrawal symptoms. Topiramate is associated with cognitive impairment and weight reduction. Weight loss is not usually a desired effect in older patients, which makes topiramate less than ideal as a first-line treatment for alcohol use disorder. Short- and longterm cognitive dysfunction can be seen even at low doses [ 48 , 49].

\section{Maintenance Treatment for Alcohol Use Disorders: Non-pharmacologic Interventions}

Non-pharmacologic interventions for AUD are also important to consider. These may include continuing care interventions such as brief telephone counseling or telephone recovery check-ups done by either the provider or other clinic staff. With the patient's permission, keeping in contact with caregiver(s) can be an invaluable resource as caregivers are often first to notice "red flags" indicating return to use and can offer important details about the patient's recovery [14•].

Connecting patients to social support is also key to recovery from alcohol use disorder. Older adults have better longterm outcomes when their social supports promote abstinence $[50,51]$. Sources of social support include family and friends, religious or spiritual groups, and mutual-help groups. Mutualhelp programs can include more structured programs such as Alcoholics Anonymous (AA) which help connect patients to a network of peers to whom they can relate [14•]. For patients seeking an alternative to AA, Self-Management and Recovery Training (SMART) differs from AA in that it is run by trained volunteers and is based on principles of cognitive behavioral therapy (CBT) and motivational interviewing (MI). Some of the CBT and MI skills include building and keeping up motivation, coping with urges, managing thoughts, feelings, and behaviors, and living a balanced life [14•]. During the COVID-19 pandemic, mutual-help groups are more likely to be occurring through "virtual" platforms such as video or telephone which can be an advantage for patients needing to physically distance or if transportation is a barrier. However, some older adults may lack the necessary technology.

\section{Conclusion}

Substance use disorders are increasing among older adults, with alcohol being the most commonly used. It is imperative that healthcare providers appropriately screen, evaluate, and treat AUD given that older adults are more susceptible to medical and neurological complications and significant functional decline related to the long-term consequences of alcohol use. There is not a "one size fits all" treatment plan for AUD in older adults, but pharmacologic and nonpharmacologic interventions can be implemented.

\section{References}

Papers of particular interest, published recently, have been highlighted as:

- Of importance

1. Center for Behavioral Health Statistics and Quality (2020). Results from the 2019 National Survey on drug use and health: detailed tables. Rockville, MD: Substance Abuse and Mental Health Services Administration. Retrieved from www.samhsa.gov/data/

2. Grant BF, Goldstein RB, Saha TD, Chou SP, Jung J, Zhang H, et al. The epidemiology of DSM-5 alcohol use disorder: results from the National Epidemiologic Survey on alcohol and related conditions, III. JAMA Psychiatry. 2015;72:757-66.

3. Substance Abuse and Mental Health Services Administration. Improving cultural competence. Treatment improvement protocol (TIP) series 59. HHS publication no. (SMA) 14-4849: Substance Abuse and Mental Health Services Administration; 2014.

4. Yarnell S, Li L, Mac Grory B, et al. Substance Use Disorders in Later Life: A Review and Synthesis of the Literature of an Emerging Public Health Concern. The American Journal of Geriatric Psychiatry. 2020;28:226-36. This review paper provides a timely overview of the literature on substance use disorders among older adults.

5. Zahr NM, Pohl KM, Saranathan M, Sullivan EV, Pfefferbaum A. Hippocampal subfield $\mathrm{CA} 2+3$ exhibits accelerated aging in Alcohol Use Disorder: A preliminary study. Neuroimage Clin. 2019;22:101764. https://doi.org/10.1016/j.nicl.2019.101764.

6. Lehmann SW, Fingerhood M. Substance-use disorders in later life. N Engl J Med. 2018 Dec 13;379(24):2351-60. https://doi.org/10. 1056/NEJMra1805981.

7. Taylor MH, Grossberg GT (2012). The growing problem of illicit substance abuse in the elderly: a review. Primary Care Companion for CNS Disorders, 14(4).

8. Satre DD, Hirschtritt ME, Silverberg MJ, Sterling SA. Addressing Problems with Alcohol and Other Substances Among Older Adults During the COVID-19 Pandemic. Am J Geriatr Psychiatry. 2020 Jul;28(7):780-783. https://doi.org/10.1016/j.jagp.2020.04.012. Epub 2020 Apr 22. PMID: 32359882; PMCID: PMC7174977. This article provides COVID-19 specific updates to the literature on alcohol use disorder among older adults.

9. Weerakoon SM, Jetelina KK, Knell G. Longer time spent at home during COVID-19 pandemic is associated with binge drinking among US adults. Am J Drug Alcohol Abuse. 2020 Dec;7:1-9. https://doi.org/10.1080/00952990.2020.1832508 Epub ahead of print.

10. Daly M, Robinson E. High-Risk Drinking in Midlife Before Versus During the COVID-19 Crisis: Longitudinal Evidence From the United Kingdom. Am J Prev Med. 2020 Nov 21:S07493797(20)30421-9. https://doi.org/10.1016/j.amepre.2020.09.004. Epub ahead of print. PMID: 33234355; PMCID: PMC7680033.

11. Khatri UG, Perrone J. Opioid Use Disorder and COVID-19: Crashing of the Crises. J Addict Med. 2020 Jul/Aug;14(4):e6-e7. 
https://doi.org/10.1097/ADM.0000000000000684. PMID: 32404651 ; PMCID: PMC7236857.

12. Office of the Surgeon General. Facing addiction in America: the surgeon General's report on alcohol, drugs, and health: Department of Health and Human Services; 2016.

13. Schulte MT, Hser YI. Substance use and associated health conditions throughout the lifespan. Public Health Rev. 2014;35(2):ii.

14. Substance Abuse and Mental Health Services Administration. Treating Substance Use Disorder in Older Adults. Treatment Improvement Protocol (TIP) Series 26. HHS Publication No. PEP20-02-01-011: Substance Abuse and Mental Health Services Administration; 2020. This highly important reference includes SAMHSA's 2020 recommendations regarding evaluation and management of various substance use disorders among older adults.

15. U.S. Preventive Services Task Force. (2018). Final recommendation statement: Unhealthy alcohol use in adolescents and adults: Screening and behavioral counseling interventions.

16. Berks J, McCormick R. Screening for alcohol misuse in elderly primary care patients: a systematic literature review. Int Psychogeriatr. 2008 Dec;20(6):1090-103. https://doi.org/10.1017/ S1041610208007497 Epub 2008 Jun 9.

17. Ewing JA. Detecting alcoholism. The CAGE questionnaire JAMA. 1984 Oct 12;252(14):1905-7. https://doi.org/10.1001/jama.252.14. 1905.

18. Barbor TF, Higgins-Biddle JC, Saunders JB, Monteiro MG (2001). AUDIT: the alcohol use disorders identification test: guidelines for use in primary care (2nd ed., p. 17). Geneva, Switzerland: World Health Organization.

19. Selin KH. Test-retest reliability of the alcohol use disorder identification test in a general population sample. Alcohol Clin Exp Res. 2003;27(9):1428-35.

20. Purcell B, Olmstead MC. The performance and feasibility of three brief alcohol screening tools in a senior population. Can Psychol. 2014;55(2a):19.

21. Aalto M, Alho H, Halme JT, Seppä K. The alcohol use disorders identification test (AUDIT) and its derivatives in screening for heavy drinking among the elderly. International Journal of Geriatric Psychiatry. 2011;26(9):881-5.

22. Bush K, Kivlahan DR, McDonell MB, Fihn SD, Bradley KA. The AUDIT alcohol consumption questions (AUDIT-C): an effective brief screening test for problem drinking. Ambulatory care quality improvement project (ACQUIP). Arch Intern Med. 1998;158: 1789-95.

23. Purcell B, Flower MC, Busto U. (2017). Senior alcohol misuse Indicator (SAMI) tool. Centre for Addiction and Mental Health.

24. Blow FC, et al. Brief screening for alcohol problems in elderly populations using the short Michigan alcoholism screening testgeriatric version (SMAST-G): Research Society on Alcoholism Annual Scientific Meeting; 1998.

25. Barry KL, Blow FC. Drinking over the lifespan: focus on older adults. Alcohol Research. 2016;38(1):115-20.

26. Schonfeld L, Hazlett RW, Hedgecock DK, Duchene DM, Burns LV, Gum AM. Screening, brief intervention, and referral to treatment for older adults with substance misuse. Am J Public Health. 2015;105(1):205-11. https://doi.org/10.2105/AJPH.2013.301859.

27. National Institute on Alcohol Abuse and Alcoholism. Older Adults. (2021). http:///www.niaaa.nih.gov/alcohols-effects-health/specialpopulations-co-occurring-disordered-older-adults. Accessed 2021 Apr 22.

28. Department of Defense (2017). Brief Alcohol Interventions for Unhealthy Alcohol Use. Available from: https://pdhealth.mil/ sites/default/files/images/docs/brief-alcohol-interventions-forunhealthy-alcohol-use.pdf
29. Friedrichs A, Spies M, Härter M, Buchholz A. Patient preferences and shared decision making in the treatment of substance use disorders: a systematic review of the literature. PLoS One. 2016;11(1): e0145817.

30. Schutte K, Lemke S, Moos RH, Brennan PL. Age-sensitive psychosocial treatment for older adults with substance abuse. In: Crome I, Wu L-T, Rao RT, Crome P, editors. Substance use and older people: Wiley-Blackwell; 2015. p. 314-39.

31. Kuerbis A, Sacco P. A review of existing treatments for substance abuse among the elderly and recommendations for future directions. Subst Abus. 2013;7:13-37.

32. National Guardianship Association. (2013). Standards of practice (4th ed.). Retrieved from www.guardianship. org/wp-content/ uploads/2017/07/NGA-Standards-with-Summit-Revisions-2017. pdf.

33. Wilkins JM. Dementia, decision making, and quality of life. AMA J Ethics. 2017;19:637-9.

34. Bommersbach TJ, Lapid MI, Rummans TA, Morse RM. Geriatric alcohol use disorder: a review for primary care physicians. Mayo Clin Proc. 2015;90(5):659-66.

35. Oslin DW, Zanjani F. (2016). Treatment of unhealthy alcohol use in older adults. Alcohol and aging: clinical and public health perspectives (pp. 181-199). New York, NY: springer.

36. Substance Abuse and Mental Health Services Administration and National Institute on Alcohol Abuse and Alcoholism. Medication for the treatment of alcohol use disorder: a brief guide. HHS publication no. (SMA) 15-4907: Substance Abuse and Mental Health Services Administration; 2015.

37. Winslow BT, Onysko M, Hebert M. Medications for alcohol use disorder. Am Fam Physician. 2016;93(6):457-65.

38. Sinclair J, Chambers S, Shiles C, Baldwin DS. Safety and tolerability of pharmacological treatment of alcohol dependence: comprehensive review of evidence. Drug Saf. 2016;39:627-45.

39. Mooney LJ, McCance-Katz EF. (2016). Psychopharmacological treatments. Clinical textbook of addictive disorders (4th ed., pp. 668-694). New York, NY: Guilford press.

40. Hassell C, Wilkins K, Trevisan LA. Pharmacology of geriatric substance use disorders: considerations and future directions. Current Treatment Options in Psychiatry. 2017;4(1):102-15.

41. Le Roux C, Tang Y, Drexler K. Alcohol and opioid use disorder in older adults: neglected and treatable illnesses. Current Psychiatry Reports. 2016;18:87.

42. Lal R, Pattanayak RD. Alcohol use among the elderly: issues and considerations. Journal of Geriatric Mental Health. 2017:4(1):4-10.

43. Skinner MD, Lahmek P, Pham H, Aubin HJ. Disulfiram efficacy in the treatment of alcohol dependence: a meta-analysis. PLoS One. 2014;9(2):e87366. https://doi.org/10.1371/journal.pone.0087366.

44. Witkiewitz K, Saville K, Hamreus K. Acamprosate for treatment of alcohol dependence: mechanisms, efficacy, and clinical utility. Ther Clin Risk Manag. 2012;8:45-53.

45. Puzantian T, Carlat D. Medication fact book for psychiatric practice. 3rd ed. Newburyport, Massachusetts: Carlat Publishing; 2016.

46. Mason BJ, Quello S, Goodell V, Shadan F, Kyle M, Begovic A. Gabapentin treatment for alcohol dependence: a randomized controlled trial. JAMA Intern Med. 2014 Jan 1;174(1):70-7. https:// doi.org/10.1001/jamainternmed.2013.11950.

47. Mehrizi M, Fontem RF, Gearhart TR, Pascuzzi RM. (2012). Medications and Myasthenia Gravis: A Reference for Healthcare Professionals. Available from: https://myasthenia.org/portals/0/ draft medications and myasthenia gravis for MGFA website $8 \% 2010 \% 2012 . p \mathrm{df}$

48. Jose NA, Yadav P, Kapoor A, Mahla VP. Comparison between baclofen and topiramate in alcohol dependence: a prospective study. Ind Psychiatry J. 2019;28:44-50.

49. De Sousa A, (2010), The pharmacotherapy of alcohol dependence: a state of the Art Review In: Psychopharmacology Today, 2010. 
Available from: https:/www.ncbi.nlm.nih.gov/pmc/articles/ PMC3031941/

50. Satre DD, Chi FW, Mertens JR, Weisner CM. Effects of age and life transitions on alcohol and drug treatment outcome over nine years. Journal of Studies on Alcohol and Drugs. 2012;73(3):45968.
51. Nicholson NR. A review of social isolation: an important but underassessed condition in older adults. J Prim Prev. 2012;33(23):137-52.

Publisher's Note Springer Nature remains neutral with regard to jurisdictional claims in published maps and institutional affiliations. 